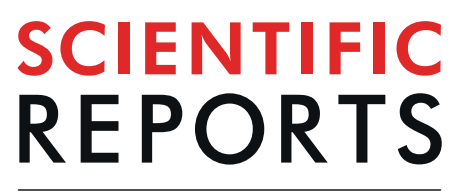

natureresearch

\title{
Photosensitive nanocarriers for specific delivery of cargo into cells
}

\author{
Pedro Mena-Giraldo $\mathbb{D}^{1}$, Sandra Pérez-Buitrago $\mathbb{D}^{2}$, Maritza Londoño-Berrío $\mathbb{D}^{3}$, \\ Isabel C. Ortiz-Trujillo ${ }^{3}$, Lina M. Hoyos-Palacio ${ }^{3} \&$ Jahir Orozco $^{1 *}$
}

\begin{abstract}
Nanoencapsulation is a rapidly expanding technology to enclose cargo into inert material at the nanoscale size, which protects cargo from degradation, improves bioavailability and allows for controlled release. Encapsulation of drugs into functional nanocarriers enhances their specificity, targeting ability, efficiency, and effectiveness. Functionality may come from cell targeting biomolecules that direct nanocarriers to a specific cell or tissue. Delivery is usually mediated by diffusion and erosion mechanisms, but in some cases, this is not sufficient to reach the expected therapeutic effects. This work reports on the development of a new photoresponsive polymeric nanocarrier (PNc)-based nanobioconjugate (NBc) for specific photo-delivery of cargo into target cells. We readily synthesized the PNcs by modification of chitosan with ultraviolet (UV)-photosensitive azobenzene molecules, with Nile red and dofetilide as cargo models to prove the encapsulation/release concept. The PNcs were further functionalized with the cardiac targeting transmembrane peptide and efficiently internalized into cardiomyocytes, as a cell line model. Intracellular cargo-release was dramatically accelerated upon a very short UV-light irradiation time. Delivering cargo in a time-space controlled fashion by means of NBcs is a promising strategy to increase the intracellular cargo concentration, to decrease dose and cargo side effects, thereby improving the effectiveness of a therapeutic regime.
\end{abstract}

Functional nanocarriers for intracellular drug delivery are systems ideally composed of biodegradable and biocompatible materials such as natural polymers, lipids, amphiphilic polymers, among others, assembled with cell-targeting biomolecules (CTBs) $)^{1,2}$. Nanocarriers can be designed for transporting a variety of cargo, either encapsulated into, adsorbed at, or dispersed with the nanocarriers ${ }^{3}$. Encapsulation of therapeutic agents into nanocarriers protects them from degradation, improves their solubility and bioavailability, and enhances the efficiency and effectiveness of therapeutic regimens. However, nanocarrier systems have shown some limitations related to storage stability and administration route, because they are susceptible to aggregation and early degradation ${ }^{4}$. Biodistribution may be also unspecific, generating inefficient therapies, side effects, genetic damage or mutations. To solve these issues, apart from carboxylic, amino, poly(ethylene glycol) and poly(phosphoester) moieties that can be placed at the outermost nanocarrier surface to confer a stealth effect, they can be functionalized with specific CTBs for site-specific controlled cargo release ${ }^{5-7}$. Targeting ability and specificity of the resultant NBcs allows them to accumulate in the target cell or tissue at higher concentrations compared to nanoparticles without activity, thus reducing doses, frequency, toxicity and potential adverse effects that most drugs intrinsically have ${ }^{8,9}$.

Whereas the small size of NBcs, which are commonly less than $200 \mathrm{~nm}$ for biomedical applications ${ }^{10,11}$, allows crossing of most physiological barriers in the body, cargo can be delivered at the specific place either by its slow diffusion or by the nanocarrier erosion mechanism. However, these naturally occurring cargo release processes may not be sufficient to reach the required doses of a certain treatment. In this context, encapsulating the cargo into PNcs for its triggered and spatial-temporal release into cells or tissues, through an external stimulus such as irradiation, could dramatically contribute to the improvement of drugs therapeutic effect ${ }^{12}$.

$\mathrm{PNcs}$ are based on polymers, which have been chemically modified to contain photoactive groups that may experience structural, conformational or stereochemical changes, such as photocleavage and photoisomerization by exposition to near-infrared (NIR) or UV irradiation ${ }^{13-16}$. Nanocarriers with irreversible photocleavable structures suffer from an alteration of their polarity by UV and NIR irradiation, producing irreversible photo-ruptures of some chromophores from the nanostructures ${ }^{17}$. In contrast, the photoactive groups of

${ }^{1}$ Max Planck Tandem Group in Nanobioengineering, Universidad de Antioquia, Calle 67 NN$^{\circ}$ 52-20, Complejo Ruta N, Medellín, 050010, Colombia. ²Departamento de Ingeniería, Pontificia Universidad Católica del Perú, Av. Universitaria 1801, Lima, Peru. ${ }^{3}$ Facultad de Medicina, Grupo de Investigación Biología de Sistemas, Universidad Pontificia Bolivariana, Calle 78 B Nº, 72A-109, Medellín, Colombia. *email: grupotandem.nanobioe@udea.edu.co 


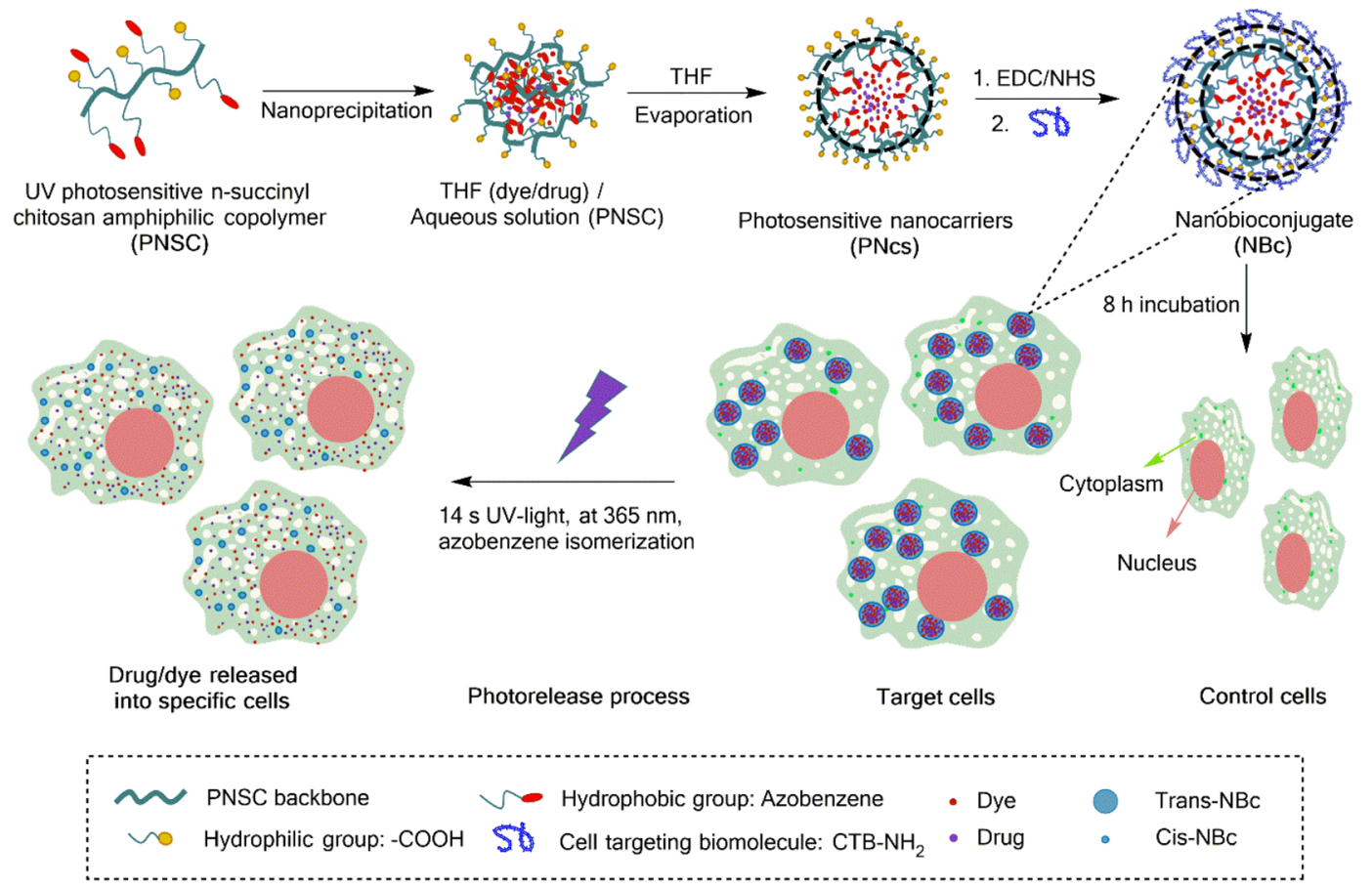

Figure 1. Schematic illustration of PNc synthesis, nanocarrier self-assembly, cargo co-encapsulation and further functionalization with the CTB. Selective internalization of the resultant $\mathrm{NBc}$ and UV-light induced cargo release into cardiomyocytes by an isomerization process of the azobenzene photosensitive moleculecontaining PNc.

reversible photoisomerization nanocarriers suffer a reversible isomerization from cis to trans by UV-absorption, where the trans isomer can be reconverted into the cis isomer by visible light ${ }^{18}$. Such rupture or isomerization processes destabilize the nanostructures, allowing the cargo to be released ${ }^{19}$. Unlike the most biocompatible NIR-based nanocarriers, whose photorelease time is in the order of hours and radiation is dispersed through the tissue ${ }^{11,20}$, the UV light-based nanocarriers, with relatively higher toxicity, require only a few minutes (or even seconds) for cargo photo-delivery ${ }^{21,22}$. In this context, the formulation of the NBcs can be fine-tuned based on the application required.

This work reports on the development of a new PNc functionalized with a CTB for rapid and specific photo-delivery of cargo into target cells (Fig. 1). The PNcs were readily synthesized by introducing UV-photosensitive azobenzene molecules into the backbone of the n-succinyl chitosan (NSC) biopolymer while monitoring all the synthesis process steps by Fourier Transform Infrared Spectroscopy (FT-IR) and Nuclear Magnetic Resonance ( ${ }^{1} \mathrm{H}$ NMR). The nanocarriers were further self-assembled and characterized by Transmission Electron Microscopy (TEM), Scanning Electron Microscopy (SEM), Dynamic Light Scattering (DLS) and Electrophoretic Light Scattering (ELS). They were further functionalized with a FITC-labelled CTB and probed to be efficiently internalized into cardiomyocytes, used as a cellular model, as demonstrated by fluorescence microscopy imaging experiments. We used Nile red and dofetilide as drug models to prove the encapsulation/release concept by estimating the intracellular cargo release by UV-vis spectrophotometry. The results show that drug release was dramatically accelerated upon a very short UV-light irradiation time. Cell line viability experiments on both incubation, with the resultant $\mathrm{NBc}$, and UV irradiation demonstrated its harmlessness at the time studied. Controlled and specific delivery of cargo by means of NBcs is a promising strategy to reach therapeutic doses with less cargo concentration, potentially decreasing side effects and improving therapy effectiveness. Indeed, the overall results demonstrated that this proof-of-concept holds considerable promise for the triggered delivery of dofetilide in cardiac cells to fight atrial fibrillation, fulfilling the demand currently requested by the scientific community for this purpose $\mathrm{e}^{23,24}$.

\section{Materials and Methods}

Materials. Nile red, 2-Chloroethanol, potassium iodide, N,N-dimethylformamide (DMF), 4-phenylazophenol, potassium carbonate, succinic anhydride, pyridine, acetic ether, hydrochloric acid $(\mathrm{HCl})$, acetic acid $\left(\mathrm{CH}_{3} \mathrm{COOH}\right)$, chitosan (medium molecular weight), acetone, sodium hydroxide $(\mathrm{NaOH})$, N, $N^{\prime}$-dicyclohexylcarbodiimide (DCC), 1-ethyl-3-(3-dimethylaminopropyl) carbodiimide (EDC), $\mathrm{N}$-hydroxysuccinimide (NHS), tetrahydrofuran (THF), isopropanol, dimethyl sulfoxide (DMSO), 2-[4-(2-hydroxyethyl)piperazin-1-yl] ethanesulfonic acid (HEPES), disodium phosphate $\left(\mathrm{Na}_{2} \mathrm{HPO}_{4}\right)$, monopotassium phosphate $\left(\mathrm{KH}_{2} \mathrm{PO}_{4}\right)$, potassium chloride $(\mathrm{KCl})$, tween 20 , sodium chloride $(\mathrm{NaCl}), 3$ - $(4,5$-dimethylthiazol-2-yl)-2,5-diphenyltetrazolium bromide (MTT), ethidium bromide, 3,3'-dihexyloxacarbocyanine iodide (DIOC6), DMEM supplemented with 10\% (v/v) fetal bovine serum. All reagents were commercially acquired 

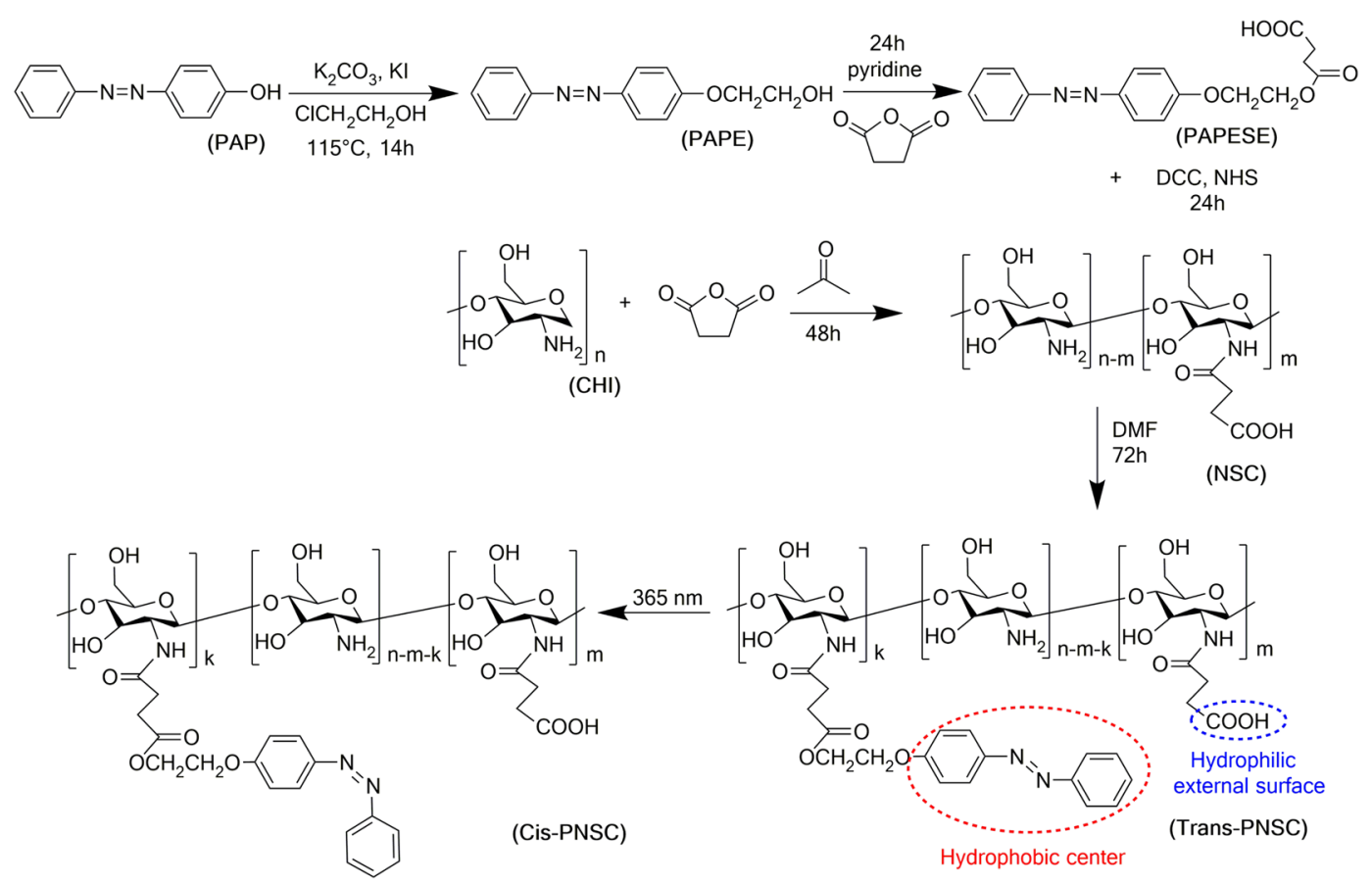

Figure 2. Synthesis and photoisomerization process of PNSC amphiphilic copolymer.

from Sigma-Aldrich (USA). Dofetiled was adquired from Cayman Chemical company. FITC-Cardiac Targeting Peptide (FITC-CTP), sequence: APWHLSSQYSRT, was commercially acquired from the GenScript Company (China). The human fetal liver cells (Hepg2), lung cells (A549) and ventricular cardiomyocytes (RL-14) are commercially available cell lines (American Type Cell Culture Patent Deposit Designation No. PTA-1499, Manassas, VA). The cellular line RL-14 has been established from nonproliferating primary cultures derived from human fetal heart tissues. They used a mitochondrial function-based method to introduce indirectly the SV-40 gene into cells, which are normally intractable to standard transformation techniques ${ }^{25,26}$.

Photosensitive amphiphilic copolymer synthesis. Synthesis of 2-(4-phenylazophenoxy)ethanol (PAPE). The azobenzene moiety PAPE was obtained by modifying the methodology published by L. Wu et al. ${ }^{27}$ A Williamson ether synthesis was carried out in two stages: First, 2-choloroethanol (1.2 ml) and potassium iodide $(2.4 \mathrm{~g})$ as catalysts were dissolved in DMF $(30 \mathrm{ml})$ under constant stirring at $70^{\circ} \mathrm{C}$ for $20 \mathrm{~min}$. Second, 4-phenylazophenol (PAP) ( $1.2 \mathrm{~g}$ ) and potassium carbonate $(5.7 \mathrm{~g})$ catalyst were added and the reaction mixture was stirred at $115^{\circ} \mathrm{C}$ for $14 \mathrm{~h}$ (Fig. 2), under reflux and nitrogen atmosphere. Finally, the catalysts were filtered and the liquid solution was stored to continue the functionalization of the polymer ${ }^{28}$.

Synthesis of photoresponsive 2-(4-(phenylazo)phenoxy)ethanoloxy succinyl ester (PAPESE). PAPE (0.2076g) and succinic anhydride $(0.6204 \mathrm{~g})$ were added to pyridine $(10 \mathrm{ml})$ and reacted in dark by $24 \mathrm{~h}$ under constant stirring at room temperature (Fig. 2). Upon completion, the reaction mixture was extracted with acetic ether/HCl removing pyridine from the reaction medium, achieved by several extraction steps. The organic phase was washed with $\mathrm{HCl}(1 \mathrm{M})$ for three times to remove pyridine. After that, the mixture was evaporated under vacuum in two stages, first at $300 \mathrm{mbar}, 60^{\circ} \mathrm{C}$ for $6 \mathrm{~h}$, then the temperature was increased to $130^{\circ} \mathrm{C}$. Finally, the solid PAPESE was removed with a plastic spatula and left in a desiccator for two days.

Synthesis of N succinyl chitosan (NSC) and N-succinyl-N-4-(2-(4-(phenylazo)phenoxy)ethanoloxy)-succinyl-chitosan (PNSC). The natural polymer chitosan (medium molecular weight, $1 \mathrm{~g}$ ) and succinic anhydride (3g) were dissolved in acetone $(20 \mathrm{ml}$ ) and reacted for $48 \mathrm{~h}$ at room temperature (Fig. 2). After that, the resultant mixture was precipitated with $4 \%(\mathrm{w} / \mathrm{v}) \mathrm{NaOH}(40 \mathrm{ml})$ and then filtered. The precipitate (NSC) was washed with ethanol three times and then dried under vacuum at $60^{\circ} \mathrm{C}$, for $6 \mathrm{~h}^{17}$. The activation of the carboxylic acid was carried out by dissolving PAPESE $(1 \mathrm{~g})$ in DMF $(10 \mathrm{ml})$ and allowing to react with DCC/NHS $(1.5 / 1.5)$ during $24 \mathrm{~h}$ in dark, under constant stirring. Subsequently, NSC $(1 \mathrm{~g})$ solubilized in $5 \mathrm{ml}$ of $\mathrm{CH}_{3} \mathrm{COOH} 0.4 \mathrm{M}$ was added to the activated-PAPESE mixture and stirred at room temperature for $72 \mathrm{~h}$ (Fig. 2 ). The copolymer was obtained by precipitation in $\mathrm{NaOH} 5 \%(w / v)$ and collected by filtration, and washed with ethanol three times. Finally, the copolymer PNSC was dried at $60^{\circ} \mathrm{C}$, for $12 \mathrm{~h}$.

Characterization and detailed spectral data of the PNSC synthetic route by ${ }^{1} H$ NMR, FT-IR and UV/Vis. PAP: ${ }^{1} \mathrm{H}$ NMR $(300 \mathrm{MHz}$, DMSO-D $): \delta 7.82(\mathrm{~d}, 4 \mathrm{H}), \delta 7.56(\mathrm{~m}, 3 \mathrm{H}), \delta 6.96(\mathrm{~d}, 2 \mathrm{H})$ from phenyl group (Fig. S1A,a, Supporting Information, S.I.); IR (KBr): $3139 \mathrm{~cm}^{-1}(-\mathrm{OH})^{\mathbf{P 1 0}}, 3060 \mathrm{~cm}^{-1}$ (phenyl group) ${ }^{\mathbf{P 1 1}}, 1606,1587,1505 \mathrm{~cm}^{-1}$ (benzene ring) $)^{\mathbf{P 1 2}}$, and $1242 \mathrm{~cm}^{-1}(-\mathrm{C}-\mathrm{O}-)^{\mathrm{P} 13}$ (Fig. 3A-PAP); UV/Vis: $\lambda_{\max } 356 \mathrm{~nm}$ (Fig. S2A, S.I. $)^{27}$. 

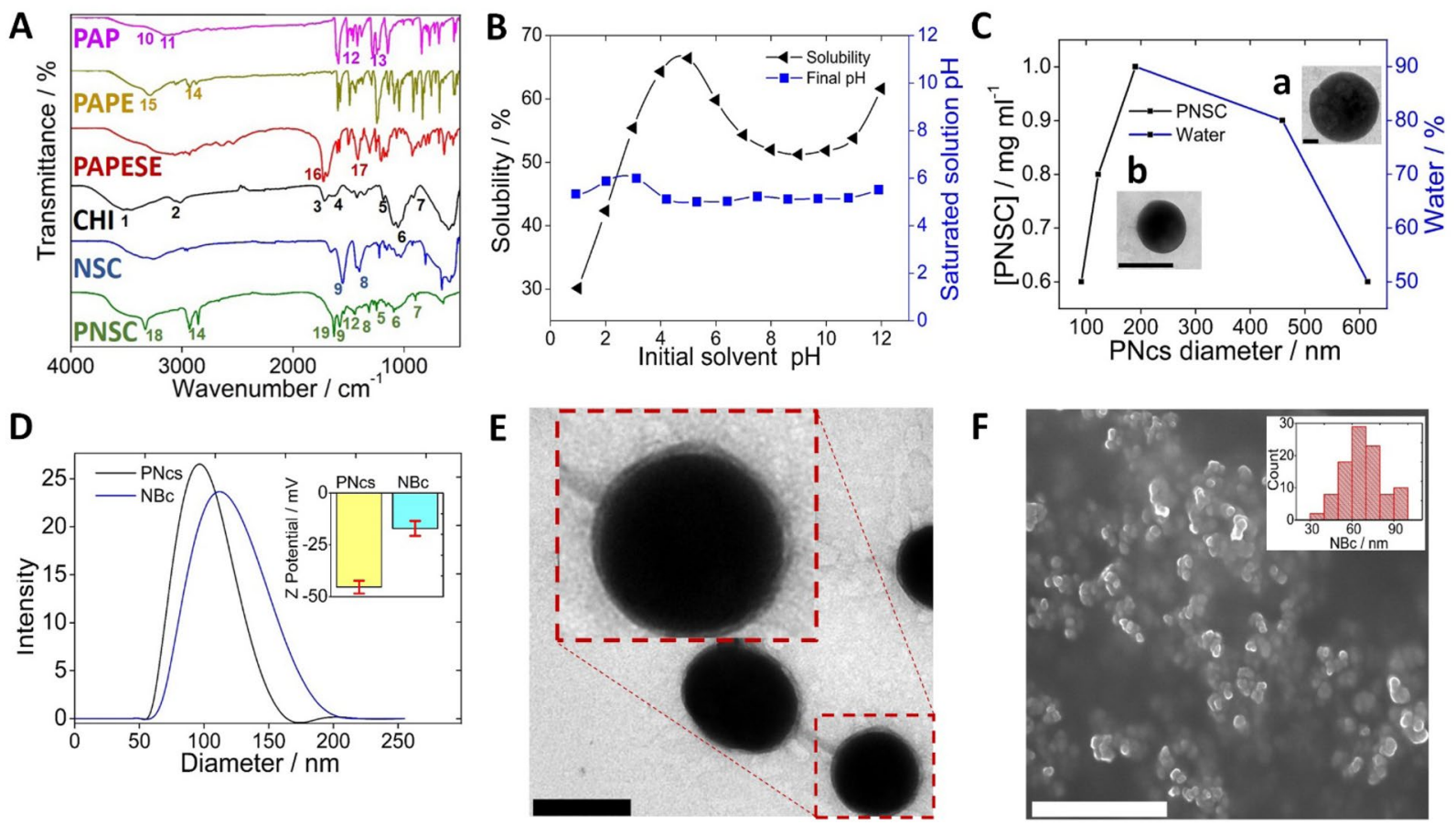

Figure 3. Characterization of the PNSC synthetic route by FT-IR (A), the characteristic peaks of FT-IR are highlighted in the text with the symbol P (for peak) as a super index. Solubility profile of PNSC in aqueous solution (B). Process of PNcs size tuning in a range of 90-615 nm (C). Inset, TEM image of PNc of $90 \mathrm{~nm}(\mathrm{a})$ and $615 \mathrm{~nm}$ (b) diameter. Estimation of PNcs and NBc particle size by DLS (D), and surface charge by ELS (D, inset), respectively. TEM image of $\mathrm{NBc}(\mathbf{E})$ and enlarged image (E, inset; dotted line). SEM image of lyophilized $\mathrm{NBc}(\mathbf{F})$ and corresponding size distribution (F, inset). The scale bars are $100 \mathrm{~nm}$ (black) and $1 \mu \mathrm{m}$ (white), respectively.

PAPE: ${ }^{1} \mathrm{H}$ NMR $\left(300 \mathrm{MHz}, \mathrm{DMSO}_{-} \mathrm{D}_{6}\right): \delta 7.91,(\mathrm{t}, 3 \mathrm{H}), \delta 7.52(\mathrm{~m}, 4 \mathrm{H}), \delta 6.85(\mathrm{~d}, 2 \mathrm{H})$ from rings of PAP (Fig. S1B,a, S.I.), $\delta 4.12$ (t,2 H), $\delta 4.08$ ppm (t,2 H), from - $\mathrm{CH}_{2^{-}}$(Fig. S1B,b, S.I.); IR (KBr): $3293 \mathrm{~cm}^{-1}(-\mathrm{OH})$, $3062 \mathrm{~cm}^{-1}$ (phenyl group), 2938, $2872 \mathrm{~cm}^{-1}(\mathrm{C}-\mathrm{H}), 1601,1582,1497 \mathrm{~cm}^{-1}$ (benzene ring) (Fig. 3A-PAPE); UV/ Vis: $\lambda_{\max } 353 \mathrm{~nm}$ (Fig. S2B, S.I. ${ }^{27}$.

PAPESE: ${ }^{1} \mathrm{H}$ NMR $(300 \mathrm{MHz}$, DMSO-D 6$): \delta 7.91(\mathrm{t}, 3 \mathrm{H}), \delta 7.53(\mathrm{~m}, 4 \mathrm{H}), \delta 6.86(\mathrm{~d}, 2 \mathrm{H})$ from rings of PAPE (Fig. S1C,a, S.I.); $\delta 4.39(\mathrm{t}, 2 \mathrm{H}), \delta 4.31(\mathrm{t}, 2 \mathrm{H})$, from $-\mathrm{CH}_{2}$ ( (Fig. S1C,b, S.I.), $\delta 2.50(2 \mathrm{H})$ from $-\mathrm{CH}_{2} \mathrm{COOH}$ (Fig. S1C,c, S.I.); IR (KBr): $3062 \mathrm{~cm}^{-1}$ (phenyl group), 2931, $2880 \mathrm{~cm}^{-1}(\mathrm{C}-\mathrm{H}), 1600,1584,1498 \mathrm{~cm}^{-1}$ (benzene ring), $1723 \mathrm{~cm}^{-1}$ (-CO-), 1417, $1384 \mathrm{~cm}^{-1}\left(-\mathrm{COO}^{-}\right.$ions) ${ }^{29}$ (Fig. $3 \mathrm{~A}-\mathrm{PAPESE}$ ); UV/Vis: $\lambda_{\max } 352 \mathrm{~nm}$ (Fig. S2C, S.I.).

CHI: ${ }^{1} \mathrm{H}$ NMR $\left(300 \mathrm{MHz}, \mathrm{D}_{2} \mathrm{O}\right.$ and TFA): $\delta 4.50\left(\mathrm{H}_{7}\right)$, from $-\mathrm{NH}_{2}$ (Fig. S1D,d, S.I.), $\delta 3.04-3.58\left(\mathrm{H}_{2}, \mathrm{H}_{3}\right.$, $\mathrm{H}_{4}, \mathrm{H}_{5}$ ), from ring of chitosan (Fig. S1D,e, S.I.), $\delta 2.85\left(\mathrm{H}_{1}\right)$, from $-\mathrm{CH}_{2} \mathrm{NH}_{2}$ (Fig. S1D,f, S.I.), $\delta 2.39$ (3 H), from $\mathrm{CH}_{3}$-CO-NH- due to acetylation of chitosan (Fig. S1D,g, S.I.); IR (KBr): $3355 \mathrm{~cm}^{-1}(-\mathrm{OH})^{\mathbf{P 1}}, 2863 \mathrm{~cm}^{-1}(-\mathrm{CH}-)^{\mathbf{P 2}}$, $1645 \mathrm{~cm}^{-1}(-\mathrm{CO}-\mathrm{NH}-)^{\mathbf{P 3}} 1575 \mathrm{~cm}^{-1}\left(-\mathrm{NH}_{2}\right)^{\mathbf{P 4}}, 1150 \mathrm{~cm}^{-1}(-\mathrm{C}-\mathrm{O}-\mathrm{C}-)^{\mathbf{P 5}}, 1026 \mathrm{~cm}^{-1}(-\mathrm{C}-\mathrm{O}-)^{\mathbf{P 6}}$ and 891 (pyranoid ring $)^{\mathbf{P 7}}$ (Fig. 3A-CHI) ${ }^{17,30,31}$.

NSC: ${ }^{1} \mathrm{H}$ NMR (300 MHz, $\mathrm{D}_{2} \mathrm{O}$ and TFA): $\delta 4.3\left(\mathrm{H}_{7}\right)$, from $-\mathrm{NH}_{2}$ (Fig. S1E,d, S.I.), $\delta 3.05-3.59\left(\mathrm{H}_{2}, \mathrm{H}_{3}, \mathrm{H}_{4}, \mathrm{H}_{5}\right)$, from ring of $\mathrm{CHI}$ (Fig. S1E,e, S.I.), $\delta 2.86\left(\mathrm{H}_{1}\right)$, from $-\mathrm{CH}_{2} \mathrm{NH}_{2}$ (Fig. S1E,f, S.I.), $\delta 2.36(3 \mathrm{H})$, from $\mathrm{CH}_{3}-\mathrm{CO}-\mathrm{NH}-$,

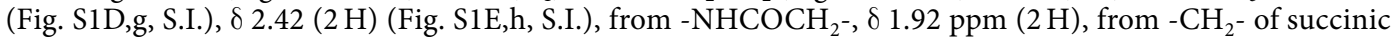
anhydride (Fig. S1E,I, S.I.); IR (KBr): $3256 \mathrm{~cm}^{-1}(-\mathrm{OH}), 2972,2947 \mathrm{~cm}^{-1}(-\mathrm{CH}-), 1657 \mathrm{~cm}^{-1}(-\mathrm{CO}-\mathrm{NH}-)$, $1552 \mathrm{~cm}^{-1}(-\mathrm{NH}-)^{\mathrm{P} 9}, 1401 \mathrm{~cm}^{-1}\left(-\mathrm{COO}^{-} \text {ions }\right)^{\mathrm{P} 8}, 1153 \mathrm{~cm}^{-1}(-\mathrm{C}-\mathrm{O}-\mathrm{C}-), 1022 \mathrm{~cm}^{-1}$ (-CH-O-) and 921 (pyranoid ring) (Fig. 3A-NSC) ${ }^{17,31}$.

PNSC: ${ }^{1} \mathrm{H}$ NMR $\left(300 \mathrm{MHz}\right.$, DMSO-D ${ }_{6}$ and TFA): $\delta 8.23$ and $(3 \mathrm{H}), \delta 8.12(4 \mathrm{H}), \delta 7.94(2 \mathrm{H})$ from rings of PAPESE (Fig. S1F,a, S.I.), $\delta 3.32-3.64\left(\mathrm{H}_{2}, \mathrm{H}_{3}, \mathrm{H}_{4}, \mathrm{H}_{5}\right)$, from ring of CHI (Fig. S1F,e, S.I.); $\delta 2.88 \mathrm{ppm}\left(\mathrm{H}_{1}\right)$, from $-\mathrm{CH}_{2} \mathrm{NH}_{2}$ (Fig. S1F,f, S.I.), $\delta 2.41(2 \mathrm{H})$ (Fig. S1F,h, S.I.), from $-\mathrm{NHCOCH}_{2^{-}}$, and $\delta 1.89(2 \mathrm{H})$ (Fig. S1F,I, S.I.), from $-\mathrm{CH}_{2}$ - of succinic anhydride; IR (KBr): $3326 \mathrm{~cm}^{-1}(-\mathrm{NHCO}-)^{\mathbf{P 1 8}}, 2936,2878 \mathrm{~cm}^{-1}(\mathrm{C}-\mathrm{H})^{\mathbf{P 1 4}}, 1630 \mathrm{~cm}^{-1}(-\mathrm{C}-\mathrm{O}-)^{\mathbf{P 1 9}}$, $1577 \mathrm{~cm}^{-1}(-\mathrm{NH}-)^{\mathrm{P} 9}, 1382 \mathrm{~cm}^{-1}\left(-\mathrm{COO}^{-} \text {ions }\right)^{\mathbf{P 8}}, 1600,1588,1495 \mathrm{~cm}^{-1}$ (benzene ring) $)^{\mathbf{P} 12}, 1147 \mathrm{~cm}^{-1}(-\mathrm{C}-\mathrm{O}-\mathrm{C}-$ )$^{\mathbf{P 5}}, 1026 \mathrm{~cm}^{-1}(-\mathrm{CO}-)^{\mathbf{P 6}}$ and $918 \mathrm{~cm}^{-1}$ (pyranoid ring) ${ }^{\mathbf{P 7}}$ (Fig. 3A-PNSC); UV/Vis: $\lambda_{\max } 331 \mathrm{~nm}$ (Fig. S2D, S.I.).

Synthesis of loaded-nanobioconjugate. PNSC solubility test. Solutions of equal concentrations of PNSC (10 $\left.\mathrm{mgml}^{-1}\right)$ were prepared at twelve different pHs (1-12): solid samples of PNSC (200 mg) were added into flasks containing distilled water $(20 \mathrm{ml}), \mathrm{pH}$ was adjusted previously with aqueous $\mathrm{HCl}$ and $\mathrm{NaOH}$ and the samples were placed under agitation during $24 \mathrm{~h}$. After that, 12 pieces of filter paper were weighted and the solution filtered through vacuum by slowly adding the mixture into a Buchner Funnel. The filter paper was removed 
and the volume of the separated liquid measured. Filters were placed on top of Petri dishes and dried at $60^{\circ} \mathrm{C}$ overnight. After that, dried filters were weighed to establish their final mass. The final $\mathrm{pH}$ was also measured. We used the equation S1, (S.I.) to obtain the saturation concentration of PNSC in the water at different pHs.

Assembly of photosensitive nanocarriers. First, a homogeneous stock of PNSC $\left(2 \mathrm{mgml}^{-1}\right)$ in deionized water was made. The $\mathrm{pH}$ level of the water was the one in which higher solubility was obtained in the previous test. A series of solutions with various concentrations $\left(0.6,0.8\right.$ and $\left.1.0 \mathrm{mgml}^{-1}\right)$ was then obtained by diluting the stock solution with water at the same $\mathrm{pH}$. Then, THF (v/v, 1:1, PNSC solution: THF) was added dropwise and slowly. Subsequently, an aqueous solution of water and THF was dripped and the effect of water/THF proportions: 50/50; $80 / 20$ and $90 / 10 \%$ v/v were evaluated. Finally, the PNcs were obtained by slow evaporation of the THF (Fig. S3, S.I.) The PNcs were washed three times by centrifugation at $5000 \mathrm{rpm}$ for $15 \mathrm{~min}$, resuspended in deionized water and stored at $4{ }^{\circ} \mathrm{C}$. The cardiac targeting peptide (CTP) has been selected herein as the cell-targeting biomolecule of the $\mathrm{NBc}$ for its demonstrated capacity to disrupt the cell membrane from the cardiac cells. The NBc was obtained by functionalizing the PNcs with the CTP by modifying the methodology published by Sehgal ${ }^{32}$ and Lu (2010) as follow ${ }^{33}$. EDC and NHS were used to activate the carboxyl-terminal groups from the outermost PNcs surface, for conjugation with primary amines from the peptide. Such activation generates a succinimide derivate, that being a very good leaving group is spontaneously replaced by the amino moiety from the CTP. The reaction is performed in 2 steps as detailed as follows. Activation of the carboxylic groups on the surface of PNcs was achieved by incubation of $50 \mu \mathrm{l}$ of the as-prepared PNcs $\left(2 \mathrm{mgml}^{-1}\right)$ in $100 \mu \mathrm{l}$ of a phosphate buffer saline (PBS) solution prepared by diluting $1.9 \mathrm{mg}$ EDC $(20 \mathrm{mM})$ and $2.1 \mathrm{mg}$ NHS $(40 \mathrm{mM})$ in $500 \mu \mathrm{l}$ of $25 \mathrm{mM}$ HEPES buffer solution $\mathrm{pH} 6.5$, for $30 \mathrm{~min}$ and washing once with $100 \mu \mathrm{l}$ of HEPES buffer. Functionalization of the FITC-CTP with activated carboxylic groups from the PNcs, by incubation of $100 \mu \mathrm{l}$ of the activated PNcs in $0.05 \mathrm{M}$ phosphate buffer saline solution (PBS), pH 9.8, containing $250 \mu \mathrm{gml}^{-1}$ FITC-CTP, for $2 \mathrm{~h}$. Washing the functionalized PNcs with $100 \mu \mathrm{l}$ of $\mathrm{pH} 7.4, \mathrm{PBS}(1 \mathrm{x})$, containing $0.05 \%$ tween 20 and store at $4{ }^{\circ} \mathrm{C}$ until use. To perform the experiments, the stored solution is centrifuged at $5000 \mathrm{rpm}$ and resuspended in $100 \mu \mathrm{lpH} 7.4, \mathrm{PBS}(1 \mathrm{x})$ (without tween). This functionalization process is depicted in Fig. S4, (S.I.).

Concentration of PNcs and FITC-CTP into the NBc. FITC-linked CTP was used to facilitate its characterization by UV- spectrophotometry. The UV spectrum of the PNcs and FITC-CTP were recorded in order to determine the maximum absorbance wavelength. Absorbance-concentration dependence curves were recorded by spectrophotometry using a Multiskan GO UV/VIS, in a wavelength ranging from 200 to $1000 \mathrm{~nm}$. From the spectra, the maximum intensity values obtained at 343 and $492 \mathrm{~nm}$ for the PNcs and FITC-CTP, respectively were used to plot two calibration curves that show the corresponding absorbance-concentration dependence. An excess of CTP was used in the reaction to assemble the $\mathrm{NBc}$, named the initial solution. After the reaction, the remained not functionalized CTP was removed by centrifugation at $5000 \mathrm{rpm}$ for 10 minutes. Both, the resultant $\mathrm{NBC}$ and the free FITC-CTP in the supernatant were then collected. The pellet was resuspended in a known volume to quantify the concentration (Fig. S5, S.I.) by means their respective absorption-concentration equations (S2 and S3, S.I).

Co-encapsulation process of cargo (Nile red and dofetilide) into NBc. The cargo was solubilized in THF before to start the nanoprecipitation process. After that, the protocol is the same as described in the section "assembly of Photosensitive nanocarriers", using $0.6 \mathrm{mg} \mathrm{ml}^{-1}$ of the PNSC. Then, the loaded PNcs were centrifuged and purified to remove cargo excess using DMSO and dialysis. Finally, the functionalization with CTP was made as described in the section "assembly of Photosensitive nanocarriers". The non-encapsulated cargo was dissolved in DMSO to calculate the loaded concentration, through an absorbance-wavelength plot built for the co-solubilized dye-drug (Fig. S6, S.I.), using their respective absorption-concentration equations (S4 and S5, S.I).

Photo-triggered and specific delivery of cargo into cells. Cells with less than 17 passages were maintained in DMEM supplemented with $10 \%(\mathrm{v} / \mathrm{v})$ fetal bovine serum at $37^{\circ} \mathrm{C}$, in a humidified atmosphere containing $5 \% \mathrm{CO}_{2}$. Biocompatibility assays were performed when cell cultures were at $80 \%$ confluence. Initially, the PNcs (or NBc) were added as treatments, at the same time, in 9 plates with cultivated cardiomyocytes. The cell cultures were characterized by fluorescence microscopy at different cultivation times, from 5 to $14 \mathrm{~h}$ for PNcs and $5,6,7$ and $8 \mathrm{~h}$ for $\mathrm{NBc}$ (each), respectively. These experiments allowed, on one hand, to establish the minimum time that the PNcs need to be internalized in the cardiomyocytes and on the other hand the affinity of the NBc for the cardiac cells. Each image was taken 3 times for each culture, and the entire experiment was done in triplicate, so that $(\mathrm{n}=9)$. Then, $1 \mathrm{ml}$ of 6000 cells ( 12 -well microplates) were incubated and $100 \mu \mathrm{L}$ of $\mathrm{NBc}$ at $0.15 \mathrm{mg} \mathrm{ml}^{-1}$, in the determined times. After, the cells are dyed with $100 \mu \mathrm{L}$ of ethidium bromide (the cell nucleus is dyed in red color) at $0.2 \mathrm{mg} \mathrm{ml}^{-1}$ and $100 \mu \mathrm{L}$ of DIOC6 (the cytoplasm is dyed in green color) at $40 \mathrm{nM}$.

A UV-light lamp at $365 \mathrm{~nm}$ (Analytik Jena; long-wave; 6 watts; $230 \mathrm{VAC} / 50 \mathrm{~Hz}$ ) was used to expose over 15-different solutions with the NBc. Each solution was exposed from cero seconds (blank) to 14 seconds with 1 -second intervals. Then, the solutions were characterized by UV-spectrophotometry, where the NBc UV spectrum has the maximum absorbance wavelengths at 331 and $433 \mathrm{~nm}$. Then, the photoisomerization process was verified when the band at $331 \mathrm{~nm}$ decreased and the one at $443 \mathrm{~nm}$ increased. The isomerization extent was determined with the area under the curve, while the blank solution is taken as a reference point, by using the Image J program.

Loaded-NBc $\left(15.15 \pm 1.61 \mu \mathrm{M}\right.$ dye and $14.57 \pm 0.87 \mu \mathrm{M}$ of the drug in $0.15 \mathrm{mg} \mathrm{ml}^{-1}$ of $\left.\mathrm{NBc}\right)$ was internalized into 6000 cells for each well (12-well microplates), for $8 \mathrm{~h}$ incubation time. After that, they were irradiated with UV-light at $365 \mathrm{~nm}$ for 14 -s while the cargo released. Then, an excess of $\mathrm{KCl}$ solution $(0.56 \%)$ was added in each well (12-well microplates) to detach the adhered cells and to perform a cellular lysis process. Then, the cells and cargo delivered from them were centrifuged at $12000 \mathrm{rpm}$, remaining in the pellet. After taking out the cell 
medium, DMSO was added and the solution centrifuged again. The cargo staying at the supernatant due to its hydrophobic tendency was measured by UV-vis spectrophotometry and the co-encapsulated dofetilide-Nile red concentration curve (in DMSO) was used to quantify the cargo released intracellularly (Fig. S6, and equations S4 and S5, S.I.).

In-vitro cytotoxicity and phototoxicity assays. To assess the short-term cytotoxic effect of $\mathrm{NBc}$ and phototoxicity effect on RL14 cardiomyocytes, a MTT assay was conducted as an indicator of the metabolic competence of the cells ${ }^{34}$. Then, RL14 cells $\left(4 \times 10^{3}\right.$ cells/well, 96-well microplates) were incubated in $10 \mu l$ of 20 freshly prepared serial-dilutions (1:2) of $\mathrm{NBc}$ (from 2 to $0 \mathrm{mg} \mathrm{ml}^{-1}$ ), for $24 \mathrm{~h}$. In a similar experiment, the cells were incubated and exposed to UV-light from 0 to $15 \mathrm{~s}$. At the end of the incubation time (in both experiments), $10 \mu \mathrm{l}$ of MTT solution $\left(5 \mathrm{mgml}^{-1}\right)$ was added to each well. The microplates were then incubated in dark, under constant stirring, for $6 \mathrm{~h}$, at $37^{\circ} \mathrm{C} .100 \mu \mathrm{l}$ of cold isopropanol was then added to each well in order to dissolve formazan crystals, followed by gentle stirring, in a gyratory shaker, for 12 to $24 \mathrm{~h}$. After that, the optical density (OD) was measured with the Multiskan-go spectrophotometer, at a wavelength of $570 \mathrm{~nm}$. To verify the repeatability of the results, three independent experiments were performed in triplicate. Also, positive (dimethyl sulfoxide, DMSO 100\%) and negative controls (complete culture medium without cells) were also tested.

For PNSC solubility test, size, concentration, charge, morphological and microstructural characterization of nanocarriers, concentration curve of co-solubilized dofetilide and Nile red, in DMSO and fluorescence microscopy characterization, please go to the Supplementary Information section.

\section{Results and Discussion}

Synthesis and characterization of the photosensitive copolymer. Figure 1 illustrates PNc synthesis, nanocarrier self-assembly and cargo co-encapsulation. PNc synthesis consists of the introduction of the UV-photosensitive 4-phenylazophenol (PAP) molecules into the backbone of a NSC biopolymer to form the amphiphilic copolymer PNSC, which will be the responsible for cargo photo-release when assembled within the PNcs. The synthesis process is followed by self-assembly of nanocapsules via the nanoprecipitation method, through which the PNSC is solubilized in an aqueous solution and dispersed through a drip system in a hydrophobic solvent. Then, while the hydrophobic solvent is being evaporated, the hydrophobic components of the PNSC are aggregated and PNcs formed (Fig. 1 and Fig. S3, S.I.) ${ }^{35}$. The resultant PNcs have a core-shell micellar structure with a hydrophobic core (the PAP) and a hydrophilic surface (carboxylic groups from NSC). They have the ability to transport a hydrophobic cargo inside the core-shell and can be functionalized with biomolecules on their outermost surface ${ }^{36}$, maintaining stability when dispersed in an aqueous medium. When the PNcs are exposed to $365 \mathrm{~nm}$ UV light, they suffer a photoisomerization process by increasing the dipole moment of PAP. Therefore, as PNcs size decreases, the polarity changes from a trans-PNSC hydrophobic center to a cis-PNSC hydrophilic one (Fig. 2) ) $^{37-39}$. With this conformational change, the PNcs are destabilized and the hydrophobic cargo is released (Fig. 1) ${ }^{19}$. It is important to highlight that the PAP has been linked to poly(acryloyl chloride), to produce a UV-photosensitive amphiphilic copolymer ${ }^{27}$ and some UV-photosensitive micelles ${ }^{19}$. Moreover, UV and NIR-nanocarriers based on chitosan have been synthesized to perform photo-triggered delivery of cargo through the photocleavage mechanism ${ }^{17,21}$. Furthermore, NSC has been extensively synthesized to produce gels and nanoparticles for biomedical applications $\mathrm{s}^{40,41}$. However, to the best of our knowledge, this is the first conjugation of NSC with PAP (chromophore molecule) to synthesize a new amphiphilic copolymer (PNSC) with the ability to form PNcs for cargo release (Fig. 2).

PNSC synthesis reaction steps were characterized through FT-IR (Fig. 3A). Initially, NSC was synthesized by the reaction between chitosan (CHI) and succinic anhydride (Fig. 2). The characteristic FT-IR peaks of CHI (Fig. 3A-CHI) remains in NSC FT-IR spectrum, and the formation of new FT-IR peaks at $1401 \mathrm{~cm}^{-1}\left(-\mathrm{COO}^{-}\right.$ ions $)^{\mathbf{P 8}}$ and $1552 \mathrm{~cm}^{-1}(-\mathrm{NH}-)^{\mathbf{P 9}}$, as well as the vanishing of the peak at $1566 \mathrm{~cm}^{-1}$, indicate the proper synthesis of the NSC (Fig. 3A-NSC) ${ }^{31}$. In the next step, which links PAP with NSC, PAP was first derivatized to PAPE and later to PAPESE. For this purpose, PAPE was initially obtained by the Williamson ether synthesis, in which 2-chloroethanol is linked to PAP to distance the -OH group from the phenyl ring. Such a distance helps to keep $-\mathrm{OH}$ group reactivity, protect $-\mathrm{N}=\mathrm{N}$ - groups and preserve their photosensitivity properties (Fig. 2$)^{28}$. The characteristic peaks of PAP (Fig. 3A-PAP) remain in PAPE FT-IR spectrum. Additionally, the peaks at 2938 and $2872 \mathrm{~cm}^{-1}(-\mathrm{C}-\mathrm{H})^{\mathrm{P} 14}$ and the $-\mathrm{OH}^{\mathrm{P} 15}$ group that shifts towards $3293 \mathrm{~cm}^{-1}$ (Fig. 3A-PAPE) evidence PAPE formation $^{27}$. Later, PAPESE was obtained from PAPE by an addition reaction in which the succinic anhydride ring opens and its - $\mathrm{OH}$ end group links to PAPE through an esterification reaction, whereas its - $\mathrm{COOH}$ end group remains free to serve later for linking to NSC (Fig. 2). As a probe of the reaction, it was observed that the PAPESE FT-IR peaks are identical to those of PAPE, but the peak at $3293 \mathrm{~cm}^{-1}(-\mathrm{OH})$ vanished and two new peaks at 1723 $\mathrm{cm}^{-1}(-\mathrm{CO}-)^{\mathrm{P} 16}$ and $1417 \mathrm{~cm}^{-1}\left(-\mathrm{COO}^{-} \text {ions) }\right)^{\mathrm{P} 17}$ appeared (Fig. 3A-PAPESE). Finally, PNSC was obtained by conjugation of NSC with PAPESE, through a carbodiimide-mediated amidation (Fig. 2). PNSC shows the principal FT-IR peaks of NSC and PAPESE, and the principal peak of amidation is sharper, i.e., $3326 \mathrm{~cm}^{-1}(-\mathrm{NHCO}-)^{\mathbf{P} 18}$, $1630 \mathrm{~cm}^{-1}(-\mathrm{CO}-)^{\mathrm{P} 19}$ (Fig. 3A-PNSC). Overall, the FT-IR results demonstrated the proper synthesis of the PNSC molecule.

PNSC synthesis reaction steps were characterized through ${ }^{1} \mathrm{H}$ NMR (Fig. S1, S.I.). When comparing the ${ }^{1} \mathrm{H}$ NMR spectrum of CHI (Fig. S1D, S.I.) and NSC (Fig. S1E, S.I.) in DMSO-D 6 and TFA, the new signals at 2.371 and $1.915 \mathrm{ppm}$ are characteristics of amidation between $\mathrm{CHI}$ and succinic anhydride, demonstrating the formation of NSC ${ }^{17}$. Comparing the PAP (Fig. S1A, S.I.) and PAPE (Fig. S1B, S.I.) ${ }^{1} \mathrm{H}$ NMR spectrums, the new signals in PAPE at 4.120 and $4.08 \mathrm{ppm}$ are characteristics of $-\mathrm{CH}_{2} \mathrm{CH}_{2} \mathrm{OH}$, confirming the proper linking of PAP and 2-chloroethanol ${ }^{27}$. For formation of PAPESE (Fig. S1C, S.I.) from PAPE, the ${ }^{1} \mathrm{H}$ NMR spectrum presents a new signal, representative of the ester group at $2.503 \mathrm{ppm}$. Finally, the PNSC ${ }^{1} \mathrm{H}$ NMR spectrum (Fig. S1F, S.I.) 
presents the principal signals of PAPESE and NSC, along with the signal at $2.410 \mathrm{ppm}$, characteristics of amidation between PAPESE and NSC. The proportion of different side chains ratio of PNSC were estimated from the FT-IR absorbance of CHI, NSC and PNSC polymers (Fig. S7, S.I.) by calculating the percent of free amines (equation S7, S.I.) and using the Table S1 from S.I. and equation S8 from S.I. to determine the percentages of the other polymer segments (Table S2, S.I.), i.e. $m=10.18 \%, k=10.06 \%, n-m-k=63.17 \%$. Thus, PAPESE and succinic anhydride are in the same proportion in the PNSC (10\%) (Table S2, S.I.), which led to homogeneous micelles.

Self-assembly of the photoresponsive nanocarriers. PNcs self-assembly is expected to be more efficient when the polymer is completely solubilized. To reach maximum solubility, we studied the solubility profile of PNSC in an aqueous solution at different pHs. A gravimetric method was then employed to calculate the saturation concentration by means of the equation S1, S.I. The $\mathrm{pH}$ of the PNSC solutions was also measured for each saturation state. The transient profile at $\mathrm{pH} 5$ indicated the maximum solubility, which agrees with its buffering capacity $\mathrm{pH}^{42}$ (Fig. $3 \mathrm{~B}$ - blue line). In addition, it was verified that, at $\mathrm{pH} 5$, the highest solubility extent is shown (Fig. 3B - black line). Thus, this $\mathrm{pH}$ value was selected for further experiments. Once PNSC was solubilized, PNcs were self-assembled by the nanoprecipitation process (Fig. 1 and Fig. S3, S.I.). PNcs size can be modulated according to the organic solvent: aqueous face ratio (tetrahydrofuran: water at $\mathrm{pH} 5$ ) and the concentration of PNSC (Fig. 3C). Thus, particle size could be tailored on demand from $615 \mathrm{~nm}$ (Fig. 3C,a) to $90 \mathrm{~nm}$ (Fig. 3C,b) demonstrating the great versatility of the approach reported herein for a variety of intra- and extra-cellular applications. Size modulation has a direct effect on loading capacity, interaction with different cells, phagocytosis of different cell types, and blood circulation time. A cargo loading capacity of $0.15 \mathrm{mgml}^{-1} \mathrm{NBc}$ was estimated to be $15.15 \pm 1.61 \mu \mathrm{M}$ dye and $14.57 \pm 0.87 \mu \mathrm{M}$ drug (Fig. 1) when co-encapsulated. Moreover, the carboxylic groups at the surface of PNcs are amenable to functionalization with CTB for cellular specific photo-triggered delivery, as shown below. Thus, PNcs size and CTB can be selected according to the required application. Herein, PNcs of around $100 \mathrm{~nm}$ size are required to pass through the cellular membrane of cardiomyocytes ${ }^{43}$. PNcs were further functionalized with the CTP through an amidation reaction between the carboxyl-terminal groups from the outermost PNcs surface and the primary amines from the CTP (Fig. S4) ${ }^{32,33}$. The CTP has shown to be able to transduce cardiomyocytes rapidly, specifically and efficiently with very little uptake by other cells ${ }^{4-46}$. The average dynamic diameter of the PNcs was $91.00 \pm 4.23 \mathrm{~nm}$, which increased by about $10 \mathrm{~nm}$ after the functionalization process (Fig. 3D) but is still optimal for cardiomyocytes uptake. Zeta potential changed from $-45.4 \pm 3.1 \mathrm{mV}$ (PNcs) to $-17.1 \pm 3.6 \mathrm{mV}(\mathrm{NBc})$ after the functionalization process. The more negative zeta potential of PNcs is caused by the presence of carboxylic groups at the outermost PNcs surface which, mostly ionized, accounts for their stability in aqueous solutions. When coupled to the CTP, primary amines from these molecules make the $\mathrm{NBc}$ have a relatively more positive charge (Fig. 3D-inset).

Size, morphology, and functionalization process were studied by TEM and SEM images. Fig. S8, S.I. shows histograms of the particle size distribution from the TEM images, from which the mean particle size was estimated to be $84.06 \pm 33.06$ and $99.07 \pm 43.32 \mathrm{~nm}$ for the PNcs and NBc, respectively. Results show not only that the NBc has an apparent slightly higher size with respect to the bare nanoparticles (PNcs and NBc), but also its sizes were slightly smaller than those estimated by DLS, as expected. This apparent discrepancy is related to the fact that whereas DLS shows the hydrodynamic particle diameter, TEM allows for a direct estimation of the geometric particle diameter. In any case, functionalized NBc showed a higher size with respect to the concomitant PNcs counterparts, which indicates that the functionalization process was indeed achieved. The depicted core/shell-like resultant structure is another indication that the PNcs were properly functionalized with CTB. The darker inner part corresponds to the more concentrated core of the polymeric particle, coming from the staining adsorbed by the polymer structure, whereas the clearer thinner outer portion is the peptide. Functionalized PNcs are completely covered by a "halo" that indicates the surface coverage extent is high (Fig. 3E), as compared to the smooth surface of the bare PNcs (Fig. 3Cb). Although their size (as estimated from SEM images) was slightly lower $(67.90 \pm 13.79 \mathrm{~nm})$, which might be related to the lyophilization process needed for SEM imaging, their spherical morphology was maintained (Fig. 3F). It is important to remark that SEM imaging doesn't require metal coating because of the presence of resonant $\pi$-electrons from the azobenzene groups in the $\mathrm{NBc}$, thus demonstrating the presence of the photosensitive molecule in the NBc structure. Self-fluorescence of PNSC with a maximum absorption peak at $343 \mathrm{~nm}$ and fluoresce of FITC-CTP with a maximum at $492 \mathrm{~nm}$, along with their initial concentrations were used to estimate the PNcs and CTP extent in the NBc by means of equations S2 and S3 (S.I.). The resultant $\mathrm{NBc}$ has a concentration of $0.15 \mathrm{mgml}^{-1}$ and contains $13.65 \pm 2.45 \mu \mathrm{gml}^{-1}$ of FITC-CTP, which corresponds to $95.63 \pm 3.28 \mu \mathrm{g}$ FITC-CTP $\mathrm{mg}^{-1}$ PNcs (Fig. S5, S.I.). This concentration seems reasonable, taking into account that the CTP is a thin layer covering the surface of the PNcs, which is enough for internalization of the $\mathrm{NBc}$ in the cardiac cells, as shown below.

Specificity of the nanobioconjugate for target cells. To test the specificity of the PNcs functionalized with CTP for the target cells, the resultant $\mathrm{NBc}$ was interrogated after incubation in cardiomyocyte cultures for 5-8 h and its fluoresce area compared with 1) those from PNcs (without CTP) after an excess of $14 \mathrm{~h}$ incubation as a control of internalization efficiency and 2) those from NBc incubated in liver and lung cell cultures for $8 \mathrm{~h}$ used as a control of cellular specificity. Moreover, a design of an experiment carried out by the blocks' statistical method allowed us to evaluate the incubation times in pairs and to determine the major intracellular internalization time (the higher area occupied by PNcs) (Table S3, S.I.). Results showed that the "hours" (time of incubation variable) have statistically significant differences $\left(\mathrm{F}_{4,30}=3488.07, \mathrm{P}<0.0001\right)$ (Table S3B, S.I.); and the "blocks" and the interaction between them "hour-blocks" does not show statistically significant differences $\left(\mathrm{F}_{2,30}=0.00\right.$, $\mathrm{P}=0.996$ and $\mathrm{F}_{8,30}=0.88, \mathrm{P}=0.99$ ), respectively (Table $\mathrm{S} 3 \mathrm{~B}, \mathrm{~S}$.I.), which indicates the reproducibility of the experiment. Besides, whereas the NBc does not present internalization in the cardiac cells after a 5-h incubation time, it is slightly internalized after 6-h (Fig. 4B). This is likely a receptor-mediated cellular uptake through the cell 


\section{TRITC}

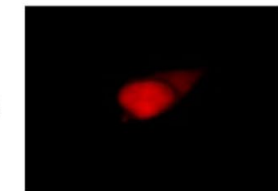

B

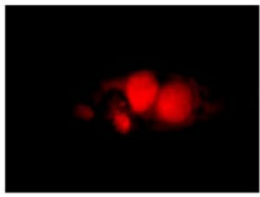

C

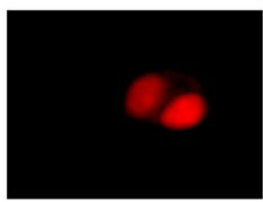

D
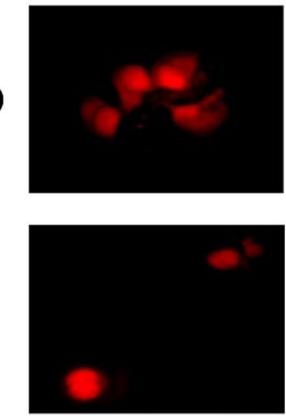

F

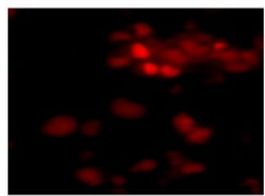

FITC
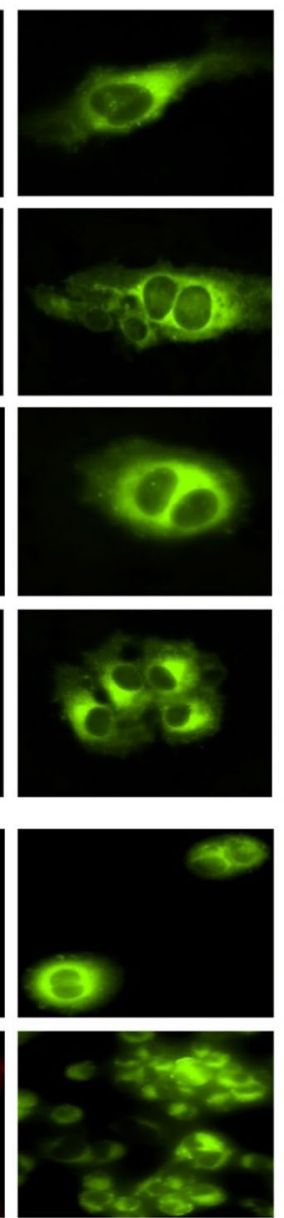

DAPI
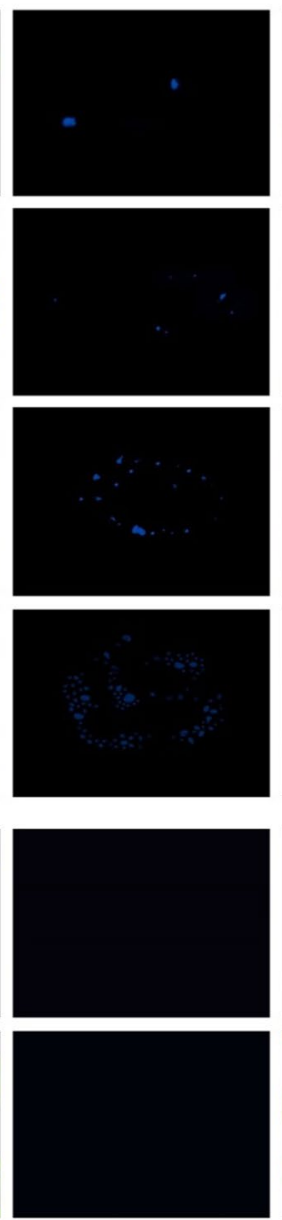

Merged
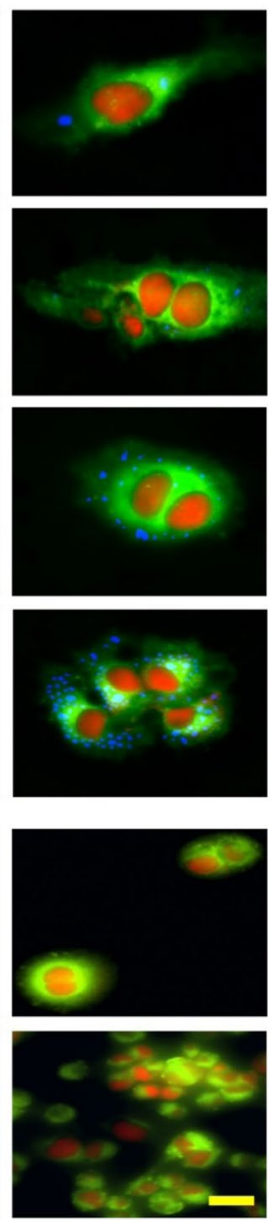

Figure 4. Fluorescence microscopy images of the cell nucleus (stained with ethidium bromide and observed by the TRITC filter), the cytoplasm (stained with FITC), the autofluorescent particles (observed by the DAPI filter) and the three of them (merged). Study the NBc internalization extent into the cardiomyocytes upon time from 6 (B), 7 (C) and 8 (D) h, with respect to the corresponding PNcs incubation for $14 \mathrm{~h} \mathrm{(A),} \mathrm{as} \mathrm{control}$ of internalization efficiency, and the negligible internalization of the NBc in liver (E) and lung (F) cells after 8 hours' incubation, as control of cell specificity. The scale is $25 \mu \mathrm{m}$.

bioreceptor-CTP interaction, whose mechanism (endocytic or non-endocytic) is still unclear ${ }^{46}$, but generates an intracellular occupied area of $0.83 \pm 0.02 \%$ (Fig. S9, S.I.). The increase of their occupied extent in the cardiac cells upon different incubation times $(6-8 \mathrm{~h})$ demonstrated the affinity of the NBc for cardiomyocytes, as compared to the internalization extent of PNcs after 14-h incubation used as control $(0.81 \pm 0.03 \%$ of occupied cellular area), (Fig. 4A and Fig. S9, S.I.). Then, 6-h of NBc incubation and 14-h of PNcs incubation are not significantly different $\left(\mathrm{t}_{30}=0.09, \mathrm{P}=0.9291\right)$ (Table S3C, S.I.). Yet, the PNcs-CTP $(\mathrm{NBc})$ uptake was 8 -h faster than that from PNcs. On the other hand, the internalization extent of the NBc slightly increased up to $1.47 \pm 0.05 \%$ and up to $15.09 \pm 0.09 \%$ after 7 and $8 \mathrm{~h}$ incubation time with respect to the control $\left(\mathrm{t}_{30}=9.12, \mathrm{P}=<0.0001\right.$ and $\mathrm{t}_{30}=93.72$, $\mathrm{P}=<0.0001$ ) (Fig. 4C,D); and Fig. S9, S.I, respectively, expected as reported by other authors for chitosan-based nanoparticles ${ }^{47}$. The specific affinity was also demonstrated when the NBc was not internalized in liver and lung cells after their incubation for 8-h (Fig. 4E,F). Moreover, the PNcs have little uptake in liver, lung and cardiac cells, at longer incubation time (14-h) (Fig. S10A,C, S.I. and Fig. 4A), and the NBc has little internalization into liver and lung cells at 14-h (Fig. S10B,D, S.I.), consistent with the literature ${ }^{44,46}$. Therefore, 8 -h of NBc incubation was the optimal internalization time selected for further experiments (Fig. 4). These results demonstrated the great affinity of $\mathrm{NBc}$ for the cardiomyocytes in comparison to the other cells. Based on that we can infer that the CTP is solely responsible for the affinity to cardiac cells and preventing uptake by the lung and liver cells, which would potentially allow a site-specific therapy to decrease the possible side effects of dofetilide on the liver, a concern reported in this kind of therapy ${ }^{48}$.

Photo-triggered delivery of cargo into target cells and toxicity studies. The next set of experiments was conducted to estimate the cargo photo-triggered release into the cardiac cells. For this purpose, we studied the evolution of the UV-vis transition bands from the PAP units (hydrophobic groups from the micelles) upon time, i.e. the signal at $331 \mathrm{~nm}$ decreasing and signal at $443 \mathrm{~nm}$ increasing (Fig. 5A), as an indication of 

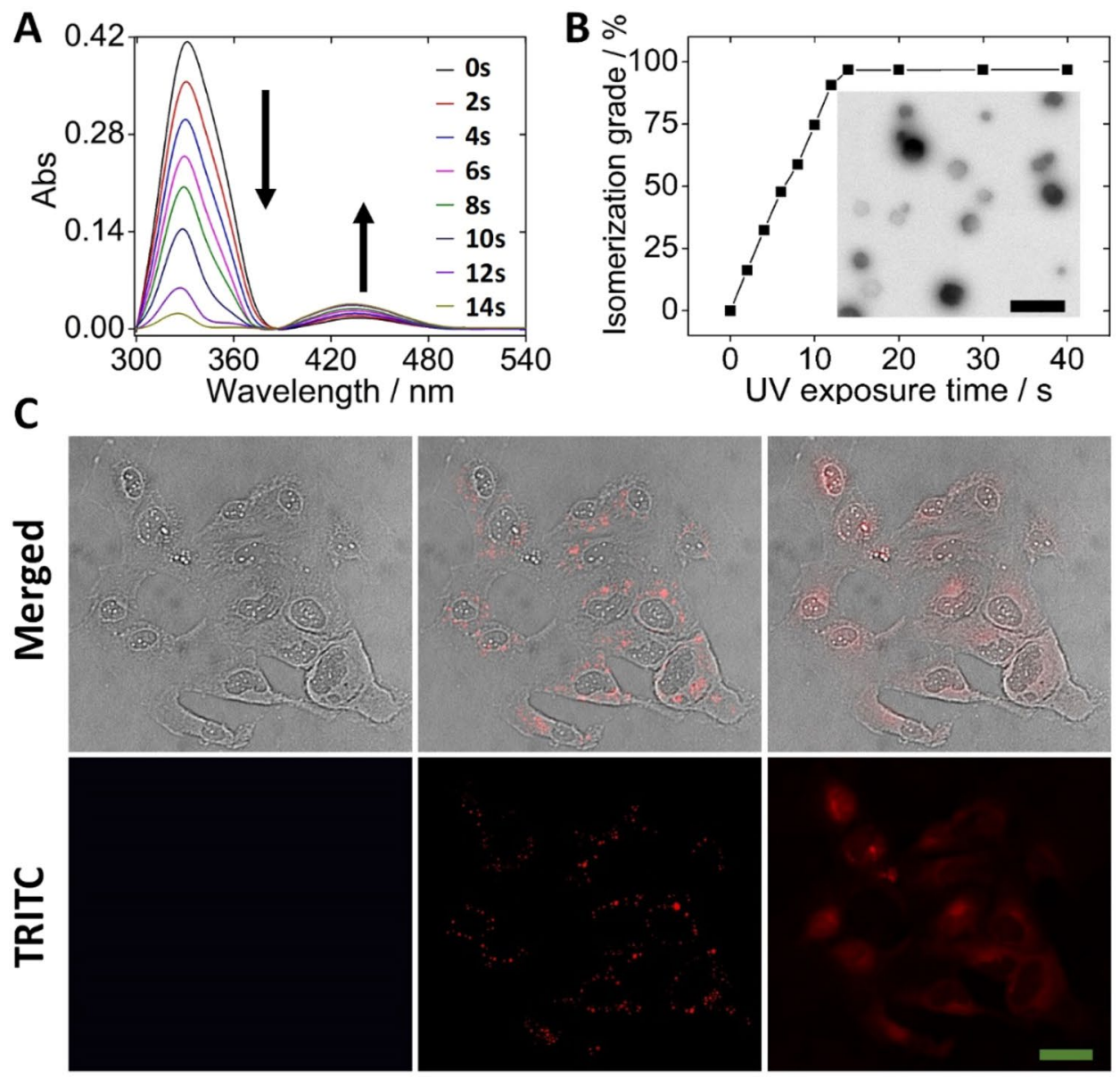

D
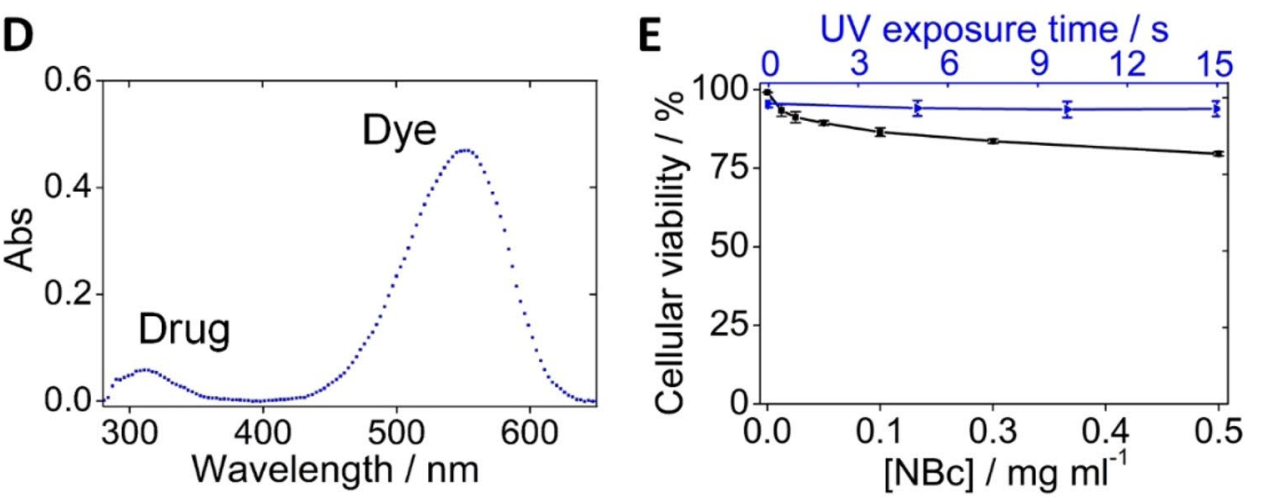

Figure 5. NBc UV-vis spectra variation induced by photoisomerization (A), NBc isomerization extent upon UV-exposure time (B) and TEM image of NBc after $14 \mathrm{~s}$ of UV-exposure (B, inset), the black scale is $100 \mathrm{~nm}$. Cargo-specific photorelease process into cardiac cells characterized by fluorescence microscopy. Untreated cells (cardiomyocytes), NBc-encapsulated cargo uptake by the cells at $8 \mathrm{~h}$ of incubation and cargo released after 14-s of UV-light irradiation at $365 \mathrm{~nm}$ are on the left, central and right columns, respectively. Upper and bottom pictures are the bright field and TRITC filter (Merged), respectively (C). The green scale is $100 \mu \mathrm{m}$. The concentration profile of co-encapsulated cargo photo-released into cells after cellular extraction (D). Cellular viability extent after 24 hours of cell uptake for the phototoxicity (blue line) and cytotoxicity (black line) tests (E).

the isomerization process. From these results, cargo photo-delivery was determined to be $97 \%$ of isomerization grade, after the NBc was exposed to UV light at $365 \mathrm{~nm}$ for 14-s (Fig. 5B). It is important to underline that the isomerization grade is directly related to the cargo-release extent (Fig. S11, S.I). Upon irradiation, the hydrophobic/hydrophilic balance of PAP from the NBc changes, making the molecules rearranges, thus destabilizing the micellar structure and releasing the cargo. Such rearranging, coming from the photoisomerization process (Fig. S12, S.I.), is responsible for the corresponding cargo release. This is clearly evidenced when comparing the TEM images from Fig. 3E with respect to those imagined after the photostimulation process (Fig. 5B, inset). The 
photo-triggered process was characterized through UV-vis spectrophotometry where the dye (Nile red) and the drug (dofetilide) have absorbance maximum peaks at $553 \mathrm{~nm}$ and $305 \mathrm{~nm}$, respectively. Moreover, results were contrasted with microscopy experiments, thanks to the dye fluorescence observed with the TRITC-filter (Fig. 5). The cargo-NBc was internalized into the cells after 8-h of incubation (optimum time established), as evidenced by the intracellular red color from the dye (Fig. 5C-center). After cells were exposed to the UV light at $365 \mathrm{~nm}$, both cargos (Nile red and dofetilide) were simultaneously delivered inside cells as inferred from the dye spread in the cytoplasm (Fig. 5C-right). Then, after the cargo was intracellularly released in 6000 cardiac cells, the concentration was estimated to be $13.82 \pm 1.47 \mu \mathrm{M}$ of the dye and $12.14 \pm 1.02 \mu \mathrm{M}$ of the drug (Fig. 5D). This concentration corresponds to $91.24 \pm 3.74 \%$ and $83.25 \pm 2.21 \%$ of the dye and drug co-encapsulated into $0.15 \mathrm{mgml}^{-1} \mathrm{NBc}_{\text {, }}$ respectively.

Cytotoxicity induced by the NBc uptake from the cardiac cell line was assessed after treating the cell cultures with six successive dilutions of $\mathrm{NBc}$ from $0 \mathrm{mgml}^{-1}$ (control) to $0.50 \mathrm{mgml}^{-1}$ for $24 \mathrm{~h}$. The cell survival plot showed a dose-dependent response trend (Fig. 5E, black line), where the NBc treatment reduced cell proliferation from $6.66 \%\left(0.016 \mathrm{mgml}^{-1}\right)$ to $20.45 \%\left(0.50 \mathrm{mgml}^{-1}\right)$, whereas the cell proliferation of untreated cells used as a control was only $0.89 \%\left(0 \mathrm{mgml}^{-1}\right)$, respectively. It was observed that cell viability slightly decreased upon increasing $\mathrm{NBcs}$ concentration. However, the NBc concentration used for the internalization experiments was only $0.15 \mathrm{mgml}^{-1}$, which showed cellular viability higher than $85 \%$. Furthermore, cell cytotoxicity was tested after $24 \mathrm{~h}$ incubation, which is much longer as compared to the $\mathrm{NBc}$ internalization time $(8 \mathrm{~h})$. Similarly, the phototoxicity of the cell line coming from the UV-light exposure at $365 \mathrm{~nm}$ was evaluated with time, with cell survival at $99 \%$ for all the studied times (Fig. $5 \mathrm{E}$ - blue line). In this context, the NBc and the UV-light would not negatively affect cell viability using the concentration $\left(0.15 \mathrm{mgml}^{-1}\right)$, incubation time $(8-\mathrm{h})$ and exposure time (14-s) selected for our experiments (Fig. 5E). Since biocompatibility of chitosan has been widely reported in the literature ${ }^{49}$ and the CTP does not represent associated toxicity at the concentration used herein, we speculate that the PAPESE molecule -containing an azo compound- may give rise to certain toxicity of the resultant $\mathrm{NBc}^{50}$. But the toxicity is minimal due to the low concentration of PAPESE in the PNSC (Table S2, S.I.).

In conclusion, new functional and biocompatible UV-photo-triggered nanocarriers for the localized, cell-specific and fast delivery of cargo have been developed. The nanocarriers were obtained through a methodology that involved the synthesis of a new polymer, self-assembly of nanocapsules and their functionalization with cell-targeting biomolecules. The developed protocol allows for the synthesis of tailor-made nanocarriers with a particle size from 90 to $615 \mathrm{~nm}$, opening a number of possibilities for a variety of applications. Moreover, this is the first PNc functionalized with a cardiac targeting peptide, for the specific photo-triggered antiarrhythmic drug delivery into cardiomyocytes, as a strategy to fight atrial fibrillation, addressing a need from scientists in this field ${ }^{23,24}$. As antiarrhythmic drugs must be delivered only to atrial cardiomyocytes to avoid the proarrhythmic and side effects, this localized therapy could be implemented through a catheter, like in the cardiac ablation-based intervention ${ }^{51,52}$. These smart nanocarriers for fast cargo delivery in specific cells pave the way for a highly specific space-temporal drug delivery triggered by external control, even in a very specific area, depending on how and where the UV-light is applied. The versatility of the PNcs allows them to be used in other types of applications, such as photodynamic therapy and skin lesions, or to treat diseases where the cargo delivery could be controlled by UV-light from sunlight.

Received: 7 November 2019; Accepted: 19 January 2020;

Published online: 07 February 2020

\section{References}

1. Karlsson, J., Vaughan, H. J. \& Green, J. J. Biodegradable Polymeric Nanoparticles for Therapeutic Cancer Treatments. Annu. Rev. Chem. Biomol. Eng. 9, 105-127 (2018).

2. Wu, Q. et al. Biocompatible and biodegradable zeolitic imidazolate framework/polydopamine nanocarriers for dual stimulus triggered tumor thermo-chemotherapy. Biomaterials 162, 132-143 (2018).

3. Wilczewska, A. Z., Niemirowicz, K., Markiewicz, K. H. \& Car, H. Nanoparticles as drug delivery systems. Pharmacol. Reports 64, 1020-1037 (2012).

4. Elsabahy, M. \& Wooley, K. L. Design of polymeric nanoparticles for biomedical delivery applications. Chem. Soc. Rev. 41, 2545-2561 (2012).

5. Kauscher, U., Holme, M. N., Björnmalm, M. \& Stevens, M. M. Physical stimuli-responsive vesicles in drug delivery: Beyond liposomes and polymersomes. Adv. Drug Deliv. Rev. 138, 259-275 (2019).

6. Juliano, R. L., Alam, R., Dixit, V. \& Kang, H. M. Cell-targeting and cell-penetrating peptides for delivery of therapeutic and imaging agents. Wiley Interdiscip. Rev. Nanomedicine. Nanobiotechnology 1, 324-335 (2009).

7. Hah, H. J. et al. Methylene blue-conjugated hydrogel nanoparticles and tumor-cell targeted photodynamic therapy. Macromol. Biosci. 11, 90-99 (2011).

8. Ruoslahti, E. Peptides as Targeting Elements and Tissue Penetration Devices for Nanoparticles. Adv. Mater. 24, 3747-3756 (2012).

9. Dasa, S. S. K. et al. Development of target-specific liposomes for delivering small molecule drugs after reperfused myocardial infarction. J. Control. Release 220, 556-567 (2015).

10. Rawat, M., Singh, D., Saraf, S. \& Saraf, S. Nanocarriers: promising vehicle for bioactive drugs. Biol. Pharm. Bull. 29, 1790-1798 (2006).

11. Zhao, T. et al. Near-Infrared Triggered Decomposition of Nanocapsules with High Tumor Accumulation and Stimuli Responsive Fast Elimination. Angew. Chemie 130, 2641-2645 (2018).

12. Sun, T. et al. Engineered nanoparticles for drug delivery in cancer therapy. Angew. Chemie Int. Ed. 53, 12320-12364 (2014).

13. Liu, Q., Zhan, C. \& Kohane, D. S. Phototriggered drug delivery using inorganic nanomaterials. Bioconjug. Chem. 28, 98-104 (2017).

14. Barhoumi, A., Liu, Q. \& Kohane, D. S. Ultraviolet light-mediated drug delivery: Principles, applications, and challenges. J. Control. Release 219, 31-42 (2015).

15. Wang, R., Li, X., Zhou, L. \& Zhang, F. Epitaxial Seeded Growth of Rare-Earth Nanocrystals with Efficient $800 \mathrm{~nm}$ Near-Infrared to $1525 \mathrm{~nm}$ Short-Wavelength Infrared Downconversion Photoluminescence for In Vivo Bioimaging. Angew. Chemie Int. Ed. 53, 12086-12090 (2014). 
16. Pérez-Buitrago, S., Mena-Giraldo, P., Pinal, R. \& Hoyos-Palacio, L. Azopolymer based nanoparticles for phototriggered drug delivery. in 201941 st Annual International Conference of the IEEE Engineering in Medicine and Biology Society (EMBC) 1089-1092 (IEEE, 2019).

17. Cao, J. et al. Near-infrared light-triggered micelles for fast controlled drug release in deep tissue. Biomaterials 34, 6272-6283 (2013).

18. Molla, M. R. et al. Dynamic actuation of glassy polymersomes through isomerization of a single azobenzene unit at the block copolymer interface. Nat. Chem 10, 659-666 (2018).

19. Li, N., Li, Y. \& Wang, X. Photoresponsive submicron-sized hollow spheres obtained from amphiphilic azobenzene-containing random copolymer. Polymer (Guildf). 53, 3975-3985 (2012).

20. Babin, J. et al. A new two-photon-sensitive block copolymer nanocarrier. Angew. Chemie 121, 3379-3382 (2009).

21. Cui, W. et al. Photosensitive nanoparticles of chitosan complex for controlled release of dye molecules. Nanotechnology 22, 65702 (2011).

22. Li, N., Li, Y. \& Wang, X. Photoresponsive submicron-sized hollow spheres obtained from amphiphilic azobenzene-containing random copolymer. Polymer (Guildf). 53, 3975-3985 (2012).

23. Avula, U. M. R. et al. Cell-specific nanoplatform-enabled photodynamic therapy for cardiac cells. Hear. Rhythm 9, 1504-1509 (2012).

24. Ravens, U. \& Odening, K. E. Atrial fibrillation: therapeutic potential of atrial K+ channel blockers. Pharmacol. Ther. 176, 13-21 (2017).

25. Maayah, Z. H., Abdelhamid, G. \& El-Kadi, A. O. S. Development of cellular hypertrophy by 8-hydroxyeicosatetraenoic acid in the human ventricular cardiomyocyte, RL-14 cell line, is implicated by MAPK and NF- $\kappa$ B. Cell Biol. Toxicol. 31, 241-259 (2015).

26. Maayah, Z. H., Elshenawy, O. H., Althurwi, H. N., Abdelhamid, G. \& El-Kadi, A. O. S. Human fetal ventricular cardiomyocyte, RL14 cell line, is a promising model to study drug metabolizing enzymes and their associated arachidonic acid metabolites. J. Pharmacol. Toxicol. Methods 71, 33-41 (2015).

27. Wu, L., Tuo, X., Cheng, H., Chen, Z. \& Wang, X. Synthesis, photoresponsive behavior, and self-assembly of poly (acrylic acid)-based azo polyelectrolytes. Macromolecules 8005-8013 (2001).

28. Tanc, M., Carta, F., Scozzafava, A. \& Supuran, C. T. 6-Substituted 1, 2-benzoxathiine-2, 2-dioxides are isoform-selective inhibitors of human carbonic anhydrases IX, XII and VA. Org. Biomol. Chem. 13, 77-80 (2015).

29. Mohanty, S., Das, B. \& Dhara, S. Poly (maleic acid)-A novel dispersant for aqueous alumina slurry. J. Asian Ceram. Soc. 1, 184-190 (2013).

30. Fernandes Queiroz, M., Melo, K., Sabry, D., Sassaki, G. \& Rocha, H. Does the use of chitosan contribute to oxalate kidney stone formation? Mar. Drugs 13, 141-158 (2015).

31. Bashir, S., Teo, Y. Y., Naeem, S., Ramesh, S. \& Ramesh, K. pH responsive N-succinyl chitosan/Poly (acrylamide-co-acrylic acid) hydrogels and in vitro release of 5-fluorouracil. PLoS One 12, e0179250 (2017).

32. Sehgal, D. \& Vijay, I. K. A Method for the High Efficiency of Water-Soluble Carbodiimide-Mediated Amidation. Anal. Biochem. 218, 87-91 (1994).

33. Lu, S. et al. Targeting of embryonic stem cells by peptide-conjugated quantum dots. PLoS One $\mathbf{5}$ (2010).

34. Bilmin, K., Kopczynska, B. \& Grieb, P. Influence of serum and albumin on the in vitro anandamide cytotoxicity toward C6 glioma cells assessed by the MTT cell viability assay: implications for the methodology of the MTT tests. Folia Neuropathol 51, 44-50 (2013).

35. Rivas, C. J. M. et al. Nanoprecipitation process: From encapsulation to drug delivery. Int. I. Pharm. 532, 66-81 (2017).

36. Nikolic, M. S. et al. Micelle and vesicle formation of amphiphilic nanoparticles. Angew. Chemie Int. Ed. 48, 2752-2754 (2009).

37. Murase, T., Sato, S. \& Fujita, M. Switching the Interior Hydrophobicity of a Self-Assembled Spherical Complex through the Photoisomerization of Confined Azobenzene Chromophores. Angew. Chemie Int. Ed. 46, 5133-5136 (2007).

38. Schimka, S. et al. Photosensitive microgels containing azobenzene surfactants of different charges. Phys. Chem. Chem. Phys. 19, 108-117 (2017).

39. Montagna, M. \& Guskova, O. Photosensitive cationic azobenzene surfactants: Thermodynamics of hydration and the complex formation with poly (methacrylic acid). Langmuir 34, 311-321 (2017).

40. Yamaguchi, R., Arai, Y., Itoh, T. \& Hirano, S. Preparation of partially N-succinylated chitosans and their cross-linked gels. Carbohydr. Res. 88, 172-175 (1981).

41. Hou, Z. et al. Synthesis and evaluation of N-succinyl-chitosan nanoparticles toward local hydroxycamptothecin delivery. Carbohydr. Polym. 81, 765-768 (2010).

42. Xu, P., Bajaj, G., Shugg, T., Van Alstine, W. G. \& Yeo, Y. Zwitterionic chitosan derivatives for pH-sensitive stealth coating. Biomacromolecules 11, 2352-2358 (2010).

43. Hickey, J. W., Luis, J., Williford, J. \& Mao, H. Control of polymeric nanoparticle size to improve therapeutic delivery. J. Control. Release, https://doi.org/10.1016/j.jconrel.2015.10.006 (2015).

44. Zahid, M. et al. Identification of a cardiac specific protein transduction domain by in Vivo biopanning using a M13 phage peptide display library in mice. PLoS One 5 (2010).

45. Zahid, M. \& Robbins, P. D. Cell-type specific penetrating peptides: therapeutic promises and challenges. Molecules 20, 13055-13070 (2015).

46. Zahid, M. et al. Cardiac Targeting Peptide, a Novel Cardiac Vector: Studies in Bio-Distribution, Imaging Application, and Mechanism of Transduction. Biomolecules 8, 147 (2018).

47. Yue, Z.-G. et al. Surface charge affects cellular uptake and intracellular trafficking of chitosan-based nanoparticles. Biomacromolecules 12, 2440-2446 (2011)

48. Rose, P. G., Seabury, R. \& Cwikla, G. Acute Hepatocellular Jaundice After Dofetilide Initiation: A Case Report. Hosp. Pharm. 53, $55-58(2018)$.

49. Cheung, R. C. F., Ng, T. B., Wong, J. H. \& Chan, W. Y. Chitosan: an update on potential biomedical and pharmaceutical applications. Mar. Drugs 13, 5156-5186 (2015).

50. Gičević, A., Hindija, L. \& Karačić, A. Toxicity of Azo Dyes in Pharmaceutical Industry. In International Conference on Medical and Biological Engineering 581-587 (Springer, 2019).

51. Dong, X., Sun, Q., Tang, M. \& Zhang, S. Catheter Ablation of Ventricular Arrhythmias Originating From the Junction of Pulmonary Sinus Cusp Via A Nonreversed U Curve Approach. Hear. Rhythm, https://doi.org/10.1016/j.hrthm.2019.04.010 (2019).

52. Ransbury, T. J., Armstrong, K. C., Amirana, O., Larson, C. \& Bowen, J. Systems and methods for determining tissue type (2019)

\section{Acknowledgements}

P.M.-G. and J.O. acknowledge financial support from Colciencias, the University of Antioquia and the Max Planck Society through the Cooperation agreement 566-1, 2014; and The Ruta N complex for hosting the Max Planck Tandem Groups. 


\section{Author contributions}

P.M.-G., S.P.-B., L.M.H.-P. and J.O. conceived and designed the research. P.M.-G., S.P.-B. and M.L-B. carried out the experiments with the supervision of I.C.O.-T., L.M.H.-P. and J.O. P.M.-G., S.P.-B. and J.O. wrote the manuscript. P. M.-G. designed and drew the figures. All authors have reviewed and approved the final version of the manuscript.

\section{Competing interests}

The authors declare no competing interests.

\section{Additional information}

Supplementary information is available for this paper at https://doi.org/10.1038/s41598-020-58865-z.

Correspondence and requests for materials should be addressed to J.O.

Reprints and permissions information is available at www.nature.com/reprints.

Publisher's note Springer Nature remains neutral with regard to jurisdictional claims in published maps and institutional affiliations.

(c) (i) Open Access This article is licensed under a Creative Commons Attribution 4.0 International License, which permits use, sharing, adaptation, distribution and reproduction in any medium or format, as long as you give appropriate credit to the original author(s) and the source, provide a link to the Creative Commons license, and indicate if changes were made. The images or other third party material in this article are included in the article's Creative Commons license, unless indicated otherwise in a credit line to the material. If material is not included in the article's Creative Commons license and your intended use is not permitted by statutory regulation or exceeds the permitted use, you will need to obtain permission directly from the copyright holder. To view a copy of this license, visit http://creativecommons.org/licenses/by/4.0/.

(C) The Author(s) 2020 